SCIENTIFIC REPORT

\title{
Normal tension glaucoma is not associated with the common apolipoprotein $\mathrm{E}$ gene polymorphisms
}

\author{
S Lake, E Liverani, M Desai, R Casson, B James, A Clark, J F Salmon
}

Br J Ophthalmol 2004;88:491-493. doi: 10.1136/bjo.2003.023366

\begin{abstract}
Background/aims: In normal tension glaucoma (NTG) factors other than raised intraocular pressure have a role in the pathogenesis of the optic neuropathy. Because particular apolipoprotein E (ApoE) gene polymorphisms have been associated with cell death and survival in neurological degenerative diseases, the purpose of this study was to determine the ApoE allele frequencies in patients with normal tension glaucoma.

Methods: The apolipoprotein E genotype of 155 patients with normal tension glaucoma was compared to that of 349 non-affected, control subjects from the same geographical area. A similar comparison was made between 53 patients with normal tension glaucoma who demonstrated progressive visual field loss, and control subjects. The frequencies of genotypes was compared with the $\chi^{2}$ test and MantelHaenszel coefficent.

Results: There was no significant difference in the frequency of ApoE alleles or genotypes in the normal tension glaucoma population compared to the control group. The ApoE alleles and genotypes in NTG patients with progressive disease were not different from the control group.

Conclusion: ApoE gene polymorphisms are not linked to normal tension glaucoma, suggesting that this gene does not have a role in the pathogenesis of optic neuropathy in this disease.
\end{abstract}

G laucoma is a degenerative optic neuropathy characterised by optic nerve head changes and visual field loss often related to raised intraocular pressure (IOP). Factors other than IOP are likely to have a role in the pathogenesis of glaucomatous optic neuropathy, particularly in individuals with normal tension glaucoma (NTG).

Neurological research has linked apolipoprotein E (ApoE) alleles to neuronal death (and survival) in cerebral ischaemia. Apolipoprotein E is an essential component of circulating lipoproteins and is a ligand for low density lipoprotein receptors. It exists as three common isoforms E2, E3, and E4, encoded by different alleles $(\varepsilon 2, \varepsilon 3, \varepsilon 4)$ on a single gene on chromosome 19. Each isoform differs by a single amino acid but these changes modify receptor binding and lipid metabolism. In individuals with acute cerebral ischaemia, such as an intracerebral haemorrhage, the $\varepsilon 4$ allele confers a much higher mortality, or poorer functional recovery if the individual survives, than the presence of the more common $\varepsilon 2$ and $\varepsilon 3$ alleles. ${ }^{1}$ Possession of the $\varepsilon 4$ allele is associated with a reduced outcome after traumatic head injury, ${ }^{23}$ and increased risk of earlier development of Alzheimer's disease. ${ }^{4}$

In the rat eye, ApoE has been demonstrated to be synthesised by Muller cells, and secreted into the vitreous, where lipoproteins are assembled. ApoE is absorbed by the ganglion cells (RGC), transported down the optic nerve, and may have a role in axonal nutrition. ${ }^{5}$ This possible role in RGC metabolism, together with its documented effect on neuronal survival following ischaemic and other insults, has led to the hypothesis that particular ApoE isoforms could be related to neuronal damage in glaucoma patients. ${ }^{6}$ The purpose of this study was to determine if common polymorphisms in the ApoE gene were associated with disease in a population of patients with normal tension glaucoma (NTG), a condition where retinal ganglion cells appear to be lost at lower intraocular pressure than is seen in primary open angle glaucoma.

\section{PATIENTS AND METHODS}

A total of 155 unrelated patients with NTG were recruited from glaucoma clinics in Oxfordshire and Buckinghamshire. Ninety subjects $(58 \%)$ were female and 65 male, with an age range of 34-90 years (mean 70 years). The study was approved by the Central Oxford Research and Stoke Mandeville Hospital ethics committees, and informed consent was received in each case.

The diagnosis of normal tension glaucoma was modelled on that used by the Collaborative Normal Tension Glaucoma Study Group. ${ }^{7}$ All subjects had glaucomatous optic neuropathy with corresponding visual field defects, and an intraocular pressure (IOP) never higher than $22 \mathrm{~mm} \mathrm{Hg}$ at any point throughout their follow up period (median 50 months). Phasing of the IOP was not routinely performed. A subgroup of 53 patients who had evidence of progressive optic neuropathy and visual loss over an average period of 50 months was identified.

A control population of 349 subjects was used. ${ }^{8}$ These were $57 \%$ female, aged 55 years (mean (SEM 18)) and from a similar geographic region to the NTG population, having no known glaucoma at the time of investigation. This group are a representative group to determine the distribution of ApoE gene polymorphisms in the local white population. Their ApoE polymorphism distribution is similar to other white populations in the literature.

ApoE genotype was determined with the investigator blind to the subjects' clinical history. Peripheral blood leucocyte DNA was extracted, and a 246 bp fragment of the ApoE gene was amplified by PCR, followed by mapping of restriction fragments obtained by digestion with Hhal, on $4 \%$ metaphor agarose gels. ${ }^{9}$ This allowed simultaneous identification of the three alleles for determination of the genotype in each patient.

Statistical analysis of frequency of ApoE genotypes and alleles was performed by $\chi^{2}$ test and determination of the Mantel-Haenszel coefficient. Power calculations suggested that significant differences in genotype and allele frequency at the $\mathrm{p}<0.05$ level would be observed with more than 150 patients. 
Table 1 Apolipoprotein E allele frequencies of normal tension glaucoma (NTG) subjects and controls

\begin{tabular}{llllll}
\hline & NTG & & & \multicolumn{2}{l}{ Controls } \\
\cline { 2 - 3 } \cline { 5 - 5 } Allele & No & Frequency & & No & Frequency \\
\hline 2 & 28 & 0.090 & 56 & 0.080 \\
3 & 229 & 0.739 & 534 & 0.765 \\
4 & 53 & 0.171 & 108 & 0.155 \\
Total & 310 & 1.000 & 698 & 1.000 \\
\hline
\end{tabular}

Table 2 Apolipoprotein E genotype of all normal tension glaucoma (NTG) subjects, NTG subjects with progressive visual field loss, and controls

\begin{tabular}{|c|c|c|c|c|c|c|}
\hline \multirow[b]{2}{*}{ Genotype } & \multicolumn{2}{|c|}{ All NTG } & \multicolumn{2}{|c|}{ Progressive NTG } & \multicolumn{2}{|c|}{ Controls } \\
\hline & No & Frequency & No & Frequency & No & Frequency \\
\hline $2 \backslash 2$ & 1 & 0.006 & 0 & 0.000 & 3 & 0.009 \\
\hline $2 \backslash 3$ & 16 & 0.103 & 6 & 0.113 & 37 & 0.106 \\
\hline $2 \backslash 4$ & 10 & 0.065 & 2 & 0.038 & 13 & 0.037 \\
\hline $3 \backslash 3$ & 91 & 0.587 & 32 & 0.604 & 208 & 0.596 \\
\hline $3 \backslash 4$ & 31 & 0.200 & 11 & 0.207 & 81 & 0.232 \\
\hline $4 \backslash 4$ & 6 & 0.039 & 2 & 0.038 & 7 & 0.02 \\
\hline Total & 155 & 1.000 & 53 & 1.000 & 349 & 1.000 \\
\hline
\end{tabular}

\section{RESULTS}

The allele frequency of NTG patients and controls is shown in table 1 and the distribution of ApoE genotype is shown in table 2. There was no significant difference in frequency of ApoE alleles or genotype between the normal tension glaucoma group and the control population. In addition, comparing those patients with progressive NTG disease to the controls revealed no association between ApoE genotype and disease progression (table 2).

\section{DISCUSSION}

It is well recognised that intraocular pressure is not the only factor responsible for optic nerve head damage in glaucoma. There is a considerable body of evidence to show that ApoE genotype affects vulnerability of neurons to ischaemia, survival and recovery after head injury, ${ }^{1}$ as well as its role in Alzheimer's disease. ${ }^{2}{ }^{10}{ }^{11}$ Our study was designed to see whether patients who had glaucomatous optic neuropathy despite normal IOP had a higher risk of developing the disease as a result of their ApoE genotype.

Criteria for the diagnosis of normal tension glaucoma were similar to those used in the Collaborative Normal Tension Glaucoma Study, ${ }^{7}$ with the exception that the cut off for IOP was $22 \mathrm{~mm} \mathrm{Hg}$ in our study, and no washout period was undertaken to re-evaluate baseline IOP. Patients were not phased before entry. We subdivided the group into those who had documented proof of progression despite normal IOP and those who did not show progression. This approach has the advantage of making no assumptions about the patients' condition before review and ensures that those that have progressed have done so during a period in which their measured intraocular pressures have remained within the normal range. The obvious consequence of this is that patients with subtle deterioration, and those that have been followed for a short period of time, may have been labelled as non-progressive when time could demonstrate otherwise.

A negative association between the $\varepsilon 4$ allele and risk of development of age related macular degeneration, ${ }^{12}{ }^{13}$ has been demonstrated, at least in familial cases with an earlier onset. ${ }^{14} \mathrm{~A}$ fourfold increase in the incidence of the homozygous $\varepsilon 2 / \varepsilon 2$ genotype in 100 Scottish retinitis pigmentosa families, and an eightfold increase in the $\varepsilon 4 / \varepsilon 4$ homozygous genotype, compared to the general population, has been observed. ${ }^{15}$ In glaucoma, the ApoE promoter gene polymorphisms have been shown to affect optic nerve damage and visual field loss. ApoE promoter and myocilin gene polymorphisms interact to affect optic nerve cupping and visual field loss in POAG. ${ }^{16}$ In addition, ApoE promoter variants incur risk for Alzheimer's disease independent of the $\varepsilon 4$ allele, suggesting ApoE expression may have a role in disease pathogenesis. ${ }^{17} 18$

Our study found no link between ApoE genotype and normal tension glaucoma. This is in contrast with the findings of Vickers et al, ${ }^{6}$ who found an association between the $\varepsilon 4$ allele and NTG in the Tasmanian population. However, their sample sizes were smaller (70 NTG and 51 controls) and the allele frequency in their control population was different from that observed in other white populations (Tasmanian $\varepsilon 2: \varepsilon 3: \varepsilon 4$ 14.7\%: 73.5\%: 11.8\%, WHO MONICA project ${ }^{19} 4.3-$ 12.4\%: $74.1-80.8 \%$ : $7-19.9 \%$, our data $8.0 \%$ : $76.5 \%$ : $15.5 \%$ ). The reason for the dissimilar findings is not clear and may reflect a regional difference in the Tasmanian ApoE genotype frequencies. The lack of an association of the common polymorphisms of the ApoE gene with NTG in a large white population suggests that this factor does not have a significant role in the pathogenesis of the optic neuropathy in NTG.

\section{ACKNOWLEDGEMENTS}

Financial support for this study came from the Oxfordshire Health Services Research fund. AC is supported by the Wellcome Trust, MD by Diabetes UK. We thank Dr J Levy for the use of ApoE genotype data from his control population for comparison.

\section{Authors' affiliations}

S Lake, R Casson, J F Salmon, Oxford Eye Hospital, Radcliffe Infirmary, Woodstock Road Oxford OX2 6HE, UK

E Liverani, M Desai, A Clark, Diabetes Research Laboratories, Oxford Centre for Diabetes, Endocrinology and Metabolism, Churchill Hospital, Oxford OX3 7ப, UK

B James, Stoke Mandeville Hospital, Mandeville Road, Aylesbury, HP21 8AL, UK 
Correspondence to: Stewart Lake, Department of Ophthalmology, Eye, ENT Centre, Queens Medical Centre, Derby Road, Nottingham NG7 2UH, UK; stewartlake@tiscali.co.uk

Accepted for publication 10 July 2003

\section{REFERENCES}

1 Roses AD, Saunders AM. ApoE, Alzheimer's disease, and recovery from brain stress. Ann NY Acad Sci 1997:826:200-12.

2 Teasdale GM, Nicoll JA, Murray G, et al. Association of apolipoprotein E polymorphism with outcome after head injury. Lancet 1997;350:1069-71.

3 Friedman G, Froom P, Sazabon L, et al. Apolipoprotein E-epsilon4 genotype predicts a poor outcome in survivors of traumatic brain injury. Neurology 1999:52:244-8.

4 Tilley L, Morgan K, Kalsheker N. Genetic risk factors in Alzheimer's disease. $J$ Clin Pathol 1998;51:293-304.

5 Amaratunga A, Abraham CR, Edwards RB, et al. Apolipoprotein E is synthesized in the retina by Muller glial cells, secreted into the vitreous, and rapidly transported into the optic nerve by retinal ganglion cells. J Biol Chem 1996;271:5628-32

6 Vickers JC, Craig JE, Stankovich J, et al. The apolipoprotein E4 gene is associated with elevated risk of normal tension glaucoma. Mol Vis 2002:8:389-93.

7 Collaborative Normal Tension Glaucoma Study Group. Comparison of glaucomatous progression between untreated patients with normal tension glaucoma and patients with therapeutically reduced intraocular pressures. Am J Ophthalmol 1998;126:487-497.

8 Hashim Y, Shepherd D, Wiltshire S, ef al. Butyrylcholinesterase $K$ variant on chromosome $3 q$ is associated with type II diabetes in white Caucasian subjects. Diabetologia 2001;44:2227-30.

9 Hixson JE, Vernier DT. Restriction isotyping of human apolipoprotein E by gene amplification and cleavage with Hha I. J Lipid Res 1990;31:545-8.
10 Corder EH, Saunders AM, Strittmatter WJ, et al. Gene dose of apolipoprotein E type 4 allele and the risk of Alzheimer's disease in late onset families. Science 1993;261:921-3.

11 Chartier-Harlin MC, Parfitt M, Legrain S, et al. Apolipoprotein E, epsilon 4 allele as a major risk factor for sporadic early and late-onset forms of Alzheimer's disease: analysis of the 19q13.2 chromosomal region. Hum Mol Genet 1994;3:569-74.

12 Klaver CC, Kliffen M, van Duijn CM, et al. Genetic association of apolipoprotein $\mathrm{E}$ with age-related macular degeneration. Am J Hum Genet 1998;63:200-6.

13 Souied EH, Benlian P, Amouyel P, et al. The epsilon4 allele of the apolipoprotein $\mathrm{E}$ gene as a potential protective factor for exudative agerelated macular degeneration. Am J Ophthalmol 1998;125:353-9.

14 Schmidt S, Saunders AM, De La Paz MA, et al. Association of the apolipoprotein $\mathrm{E}$ gene with age-related macular degeneration: possible effect modification by family history, age, and gender. Mol Vis 2000;6:287-93.

15 Huq L, McLachlan T, Hammer HM, et al. An increased incidence of apolipoprotein E2/E2 and E4/E4 in retinitis pigmentosa. Lipids 1993;28:995-8.

16 Copin B, Brezin AP, Valtot F, et al. Apolipoprotein E promoter singlenucleotide polymorphisms affect the phenotype of primary open angle glaucoma and demonstrate interaction with the myocilin gene. Am J Hum Genet 2002;70:1575-81.

17 Lambert J-C, Berr C, Pasquier F, et al. Pronounced impact of Th1/E47cs mutation compared with -491 AT mutation on neural APOE gene expression and risk of developing Alzheimer's disease. Hum Mol Genet 1998;7:1511-1516.

18 Lambert J-C, Araria-Goumidi L, Myllykangas L, et al. Contribution of APOE promoter polymorphisms to Alzheimer's disease risk. Neurology 2002;59:59-66.

19 Stengard JH, Weiss KM, Sing CF. An ecological study of association between coronary heart disease mortality rates in men and the relative frequencies of common allelic variations in the gene coding for apolipoprotein E. Hum Genet $1998 ; 103: 234-41$. 